\title{
Solitary Wave Solutions of the Boussinesq Equation and Its Improved Form
}

\author{
Reza Abazari ${ }^{1}$ and Adem Kılıçman ${ }^{2}$ \\ ${ }^{1}$ Young Researchers and Elite Club, Ardabil Branch, Islamic Azad University, P.O. Box 5616954184, Ardabil, Iran \\ ${ }^{2}$ Department of Mathematics, Institute of Mathematical Research, University Putra Malaysia (UPM), 43400 Serdang, Malaysia \\ Correspondence should be addressed to Reza Abazari; abazari-r@uma.ac.ir
}

Received 17 July 2013; Revised 5 September 2013; Accepted 6 September 2013

Academic Editor: Guo-Cheng Wu

Copyright (c) 2013 R. Abazari and A. Kılıçman. This is an open access article distributed under the Creative Commons Attribution License, which permits unrestricted use, distribution, and reproduction in any medium, provided the original work is properly cited.

\begin{abstract}
This paper presents the general case study of previous works on generalized Boussinesq equations, (Abazari, 2011) and (K1lıcman and Abazari, 2012), that focuses on the application of $\left(G^{\prime} / G\right)$-expansion method with the aid of Maple to construct more general exact solutions for the coupled Boussinesq equations. In this work, the mentioned method is applied to construct more general exact solutions of Boussinesq equation and improved Boussinesq equation, which the French scientist Joseph Valentin Boussinesq (1842-1929) described in the 1870s model equations for the propagation of long waves on the surface of water with small amplitude. Our work is motivated by the fact that the $\left(G^{\prime} / G\right)$-expansion method provides not only more general forms of solutions but also periodic, solitary waves and rational solutions. The method appears to be easier and faster by means of a symbolic computation.
\end{abstract}

\section{Introduction}

In the recent five decades, a new direction related to the investigation of nonlinear evolution equations (NLEEs) and processes has been actively developing in various areas of sciences. Nonlinear evolution equations have been the important subject of study in various branches of mathematicalphysical sciences such as physics, fluid mechanics, and chemistry. The analytical solutions of NLEEs are of fundamental importance, since many of mathematical-physical models are described by NLEEs. Among the possible solutions to NLEEs, certain special form solutions may depend only on a single combination of variables such as solitons. In mathematics and physics, a soliton is a self reinforcing solitary wave, a wave packet or pulse, that maintains its shape while it travels at constant speed. Solitons are caused by a cancelation of nonlinear and dispersive effects in the medium. The term "dispersive effects" refers to a property of certain systems where the speed of the waves varies according to frequency. Solitons arise as the solutions of a widespread class of weakly nonlinear dispersive partial differential equations describing physical systems. The soliton phenomenon was first described by John Scott Russell (1808-1882) who observed a solitary wave in the
Union Canal in Scotland. He reproduced the phenomenon in a wave tank and named it the "wave of translation" (also known as solitary wave or soliton) [1]. The soliton solutions are typically obtained by means of the inverse scattering transform [2] and be in dept their stability to the integrability of the field equations.

In fluid mechanics, the Boussinesq approximation for water waves is an approximation valid for weakly nonlinear and fairly long waves. The approximation is named after Joseph Valentin Boussinesq (1842-1929), who first derived them in response to the observation by John Scott Russell of the wave of translation $[3,4]$. According to the 1872 paper of Boussinesq, for water waves on an incompressible fluid and irrotational flow in the $(x, z)$ plane, the boundary conditions at the free surface elevation $z=\eta(x, t)$ are

$$
\begin{gathered}
\frac{\partial \eta}{\partial t}+\mathbf{v} \frac{\partial \eta}{\partial x}-\mathbf{w}=0, \\
\frac{\partial \varphi}{\partial t}+\frac{1}{2}\left(\mathbf{v}^{2}+\mathbf{w}^{2}\right)+g \eta=0,
\end{gathered}
$$

where $\mathbf{v}$ is the horizontal flow velocity component, $\mathbf{v}=$ $\partial \varphi / \partial x, \mathbf{w}$ is the vertical flow velocity component, $\mathbf{w}=\partial \varphi / \partial z$, 
and $g$ is the acceleration by gravity. Now, the Boussinesq approximation for the velocity potential $\varphi$, as given previously, is applied in these boundary conditions. Further, in the resulting equations, only the linear and quadratic terms with respect to $\eta$ and $\mathbf{v}_{b}$ are retained (with $\mathbf{v}_{b}=\partial \varphi_{b} / \partial x$ the horizontal velocity at the bed $z=-h$ ). The cubic and higher order terms are assumed to be negligible. Then, the following partial differential equations are obtained:

$$
\begin{aligned}
& \frac{\partial \eta}{\partial t}+\frac{\partial}{\partial x}\left[(h+\eta) \mathbf{v}_{b}\right]=\frac{1}{6} h^{3} \frac{\partial^{3} \mathbf{v}_{b}}{\partial x^{3}} \\
& \frac{\partial \mathbf{v}_{b}}{\partial t}+\mathbf{v}_{b} \frac{\partial \mathbf{v}_{b}}{\partial x}+g \frac{\partial \eta}{\partial x}=\frac{1}{2} h^{2} \frac{\partial^{3} \mathbf{v}_{b}}{\partial t \partial x^{2}} .
\end{aligned}
$$

This set of equations has been derived for a flat horizontal bed; that is, the mean depth $h$ is a constant independent of position $x$. When the right-hand sides of the previous equations are set to zero, they reduce to the shallow water equations. Under some additional approximations, but at the same order of accuracy, (2) can be reduced to a single partial differential equation for the free surface elevation $\eta(x, t)$ :

$$
\frac{\partial^{2} \eta}{\partial t^{2}}-g h \frac{\partial^{2} \eta}{\partial x^{2}}-g h \frac{\partial^{2}}{\partial x^{2}}\left(\frac{3}{2} \frac{\eta^{2}}{h}+\frac{1}{3} h^{2} \frac{\partial^{2} \eta}{\partial x^{2}}\right)=0
$$

In dimensionless quantities, by using the water depth $h$ and gravitational acceleration $g$ for nondimensionalization, (3) leads to the following, after normalization:

$$
\frac{\partial^{2} \psi}{\partial \tau^{2}}-\frac{\partial^{2} \psi}{\partial \chi^{2}}-\frac{\partial^{2}}{\partial \chi^{2}}\left(\frac{1}{2} \psi^{2}+\frac{\partial^{2} \psi}{\partial \chi^{2}}\right)=0
$$

where $\psi=3(\eta / h), \tau=\sqrt{3(g / h)}$, and $\chi=\sqrt{3}(x / h)$. In the recent years, (4) rewrites as follows [5]:

$$
u_{t t}-u_{x x}-\left(\frac{1}{2} u^{2}+q u_{x x}\right)_{x x}=0
$$

where $|q|=1$ is a real parameter. Setting $q=-1$ gives the good Boussinesq equation (GB) or well-posed Boussinesq equation, while by setting $q=1$, we get the bad Boussinesq equation (BB) or ill-posed classical Boussinesq equation. Following Bogolubsky's modification [6] in (5) when the term $q u_{x x}$, is replaced with $q u_{t t}$ it gives the so-called improved Boussinesq equation (IBq):

$$
u_{t t}-u_{x x}-\left(\frac{1}{2} u^{2}+q u_{t t}\right)_{x x}=0
$$

Similarily, using an analogous characterization used for Boussinesq equation (5), the IBq equation for $q=-1$ will give the good or well-posed (GIBq), while for $q=1$ the bad or illposed (BIBq) equation. The IBq equation appears in studying the transverse motion and nonlinearity in acoustic waves on elastic rods with circular cross-section. In particular, the BIBq is used to discuss the wave propagation at right angles to the magnetic field and also to approach the bad BS equation (see Makhankov [7]) or to study ion-sound(s) waves (see Bogolubsky [6]).
There are some review articles and some collected works that have been focused to study the classical Boussinesq equation from various points of view. The initial boundary value and the Cauchy problem of (5) have been described in [8-11]. Yajima [12] has studied the nonlinear evolution of a linearly stable solution, while the exponentially decaying solution of the spherical Boussinesq equation was obtained by Nakamura [13]. The global existence of the strong solution and the small amplitude solution for the Cauchy problem of the multidimensional equation (5) is proved in [14]. A general approach to construct exact solution to (5) is given by Clarkson [9], and Hirota [10] has deduced conservation laws and has examined $\mathrm{N}$-soliton interaction. Bona and Sachs, in [8], have discussed that the special solitary-wave solutions for (5), when nonlinear term is $u^{2}$, are nonlinearly stable for a range of their wave speeds.

On the other hand, recently, the $\left(G^{\prime} / G\right)$-expansion method, firstly introduced by Wang et al. [15], has become widely used to search for various exact solutions of NLEEs [15-19]. The value of the $\left(G^{\prime} / G\right)$-expansion method is that one treats nonlinear problems by essentially linear methods. The method is based on the explicit linearization of NLEEs for traveling waves with a certain substitution which leads to a second-order differential equation with constant coefficients. Moreover, it transforms a nonlinear equation to a simple algebraic computation. Although many efforts have been devoted to find various methods to solve (integrable or nonintegrable) NLEEs, there is no unified method. The main merits of the $\left(G^{\prime} / G\right)$-expansion method over the other methods are that it gives more general solutions with some free parameters which, by suitable choice of the parameters, turn out to be some known solutions gained by the existing methods.

Our first interest in the present work is in implementing the $\left(G^{\prime} / G\right)$-expansion method to show its power in handling nonlinear partial differential equations (PDEs), so that one can apply it to other models of various types of nonlinearity. The next interest is in the determination of exact travelling wave solutions for generalized equations (5) and (6).

\section{Description of the $\left(G^{\prime} / G\right)$-Expansion Method}

The objective of this section is to outline the use of the $\left(G^{\prime} / G\right)$ expansion method for solving certain nonlinear PDEs. Suppose that we have a nonlinear PDE for $u(x, t)$, in the form

$$
P\left(u, u_{x}, u_{t}, u_{x x}, u_{x t}, u_{t t}, \ldots\right)=0
$$

where $P$ is a polynomial in its arguments, which includes nonlinear terms and the highest order derivatives. The transformation $u(x, t)=U(\xi), \xi=k x+\omega t$, reduces (7) to the ordinary differential equation (ODE)

$$
P\left(U, k U^{\prime}, \omega U^{\prime}, k^{2} U^{\prime \prime}, k \omega U^{\prime \prime}, \omega^{2} U^{\prime \prime}, \ldots\right)=0
$$


where $U=U(\xi)$, and prime denotes derivative with respect to $\xi$. We assume that the solution of (8) can be expressed by a polynomial in $\left(G^{\prime} / G\right)$ as follows:

$$
U(\xi)=\sum_{i=1}^{m} \alpha_{i}\left(\frac{G^{\prime}}{G}\right)^{i}+\alpha_{0}, \quad \alpha_{m} \neq 0,
$$

where $\alpha_{0}$ and $\alpha_{i}$, for $i=1,2, \ldots, m$, are constants to be determined later and $G(\xi)$ satisfies a second order linear ordinary differential equation (LODE):

$$
\frac{d^{2} G(\xi)}{d \xi^{2}}+\lambda \frac{d G(\xi)}{d \xi}+\mu G(\xi)=0,
$$

where $\lambda$ and $\mu$ are arbitrary constants. Using the general solutions of (10), we have

$$
\frac{G^{\prime}(\xi)}{G(\xi)}=\left\{\begin{array}{l}
\frac{\sqrt{\lambda^{2}-4 \mu}}{2}\left(\frac{C_{1} \sinh \left(\left(\sqrt{\lambda^{2}-4 \mu} / 2\right) \xi\right)+C_{2} \cosh \left(\left(\sqrt{\lambda^{2}-4 \mu} / 2\right) \xi\right)}{C_{1} \cosh \left(\left(\sqrt{\lambda^{2}-4 \mu} / 2\right) \xi\right)+C_{2} \sinh \left(\left(\sqrt{\lambda^{2}-4 \mu} / 2\right) \xi\right)}\right)-\frac{\lambda}{2}, \quad \lambda^{2}-4 \mu>0, \\
\frac{\sqrt{4 \mu-\lambda^{2}}}{2}\left(\frac{-C_{1} \sin \left(\left(\sqrt{4 \mu-\lambda^{2}} / 2\right) \xi\right)+C_{2} \cos \left(\left(\sqrt{4 \mu-\lambda^{2}} / 2\right) \xi\right)}{C_{1} \cos \left(\left(\sqrt{4 \mu-\lambda^{2}} / 2\right) \xi\right)+C_{2} \sin \left(\left(\sqrt{4 \mu-\lambda^{2}} / 2\right) \xi\right)}\right)-\frac{\lambda}{2}, \quad \lambda^{2}-4 \mu<0,
\end{array}\right.
$$

and it follows from (9) and (10), that

$$
\begin{aligned}
U^{\prime}=-\sum_{\ell=1}^{m} \ell \alpha_{\ell} & \left(\left(\frac{G^{\prime}}{G}\right)^{\ell+1}+\lambda\left(\frac{G^{\prime}}{G}\right)^{\ell}+\mu\left(\frac{G^{\prime}}{G}\right)^{\ell-1}\right) \\
U^{\prime \prime}=\sum_{\ell=1}^{m} \ell \alpha_{\ell}( & (\ell+1)\left(\frac{G^{\prime}}{G}\right)^{\ell+2}+(2 \ell+1) \lambda\left(\frac{G^{\prime}}{G}\right)^{\ell+1} \\
& +\ell\left(\lambda^{2}+2 \mu\right)\left(\frac{G^{\prime}}{G}\right)^{\ell} \\
& +(2 \ell-1) \lambda \mu\left(\frac{G^{\prime}}{G}\right)^{\ell-1} \\
& \left.+(\ell-1) \mu^{2}\left(\frac{G^{\prime}}{G}\right)^{\ell-2}\right)
\end{aligned}
$$

and so on, here the prime denotes the derivative with respective to $\xi$. To determine $u$ explicitly, we take the following four steps.

Step 1. Determine the integer $m$ by substituting (9) along with (10) into (8) and balance the highest order nonlinear term(s) and the highest order partial derivative.

Step 2. Substitute (9) to give the value of $m$ determined in Step 1 along with (10) into (8) and collect all terms with the same order of $\left(G^{\prime} / G\right)$ together, the left-hand side of (8) is converted into a polynomial in $\left(G^{\prime} / G\right)$. Then, set each coefficient of this polynomial to zero to derive a set of algebraic equations for $k, \omega, \alpha_{0}$, and $\alpha_{i}$, for $i=1,2, \ldots, m$.

Step 3. Solve the system of algebraic equations obtained in Step 2, for $k, \omega, \alpha_{0}$, and $\alpha_{i}$, for $i=1,2, \ldots, m$, by the use of Maple.
Step 4. Use the results obtained in the above steps to derive a series of fundamental solutions $u(\xi)$ of (8) depending on $\left(G^{\prime} / G\right)$; since the solutions of (10) have been well known for us, then we can obtain exact solutions of (7).

\section{Application}

In this section, we will demonstrate the $\left(G^{\prime} / G\right)$-expansion method on three of the well-known Boussinesq type equations (5) and (6).

3.1. Boussinesq Equation. To look for the traveling wave solution of Boussinesq equation (5), we use the gauge transformation:

$$
u(x, t)=U(\xi)
$$

where $\xi=k x+\omega t$, and $k$ and $\omega$ are constants. We substitute (13) into (5) to obtain the nonlinear ordinary differential equation

$$
\left(\omega^{2}-k^{2}\right) U^{\prime \prime}-k^{2}\left(\frac{1}{2} U^{2}+q k^{2} U^{\prime \prime}\right)^{\prime \prime}=0
$$

According to Step 1, we get $m+4=2 m+2$; hence, $m=2$. We then suppose that (14) has the following formal solutions:

$$
U=\alpha_{2}\left(\frac{G^{\prime}}{G}\right)^{2}+\alpha_{1}\left(\frac{G^{\prime}}{G}\right)+\alpha_{0}, \quad \alpha_{2} \neq 0,
$$

where $\alpha_{2}, \alpha_{1}$, and $\alpha_{0}$ are constants which are unknowns to be determined later. Substituting (15) along with (10) into (14) and collecting all terms with the same order of $\left(G^{\prime} / G\right)$ together, the left-hand sides of (14) are converted into a polynomial in $\left(G^{\prime} / G\right)$. Setting each coefficient of each 
polynomial to zero, we derive a set of algebraic equations for $k, \omega, \lambda, \mu, \alpha_{0}, \alpha_{1}$, and $\alpha_{2}$ as follows:

$$
\begin{aligned}
& \left(\frac{G^{\prime}}{G}\right)^{0}:\left(-\mu \lambda\left(\lambda^{2}+8 \mu\right) \alpha_{1}-2 \mu^{2} \alpha_{2}\left(8 \mu+7 \lambda^{2}\right)\right) q k^{4} \\
& +\left(-2 \alpha_{1}^{2} \mu^{2}-\lambda \alpha_{1}\left(1+2 \alpha_{0}\right) \mu-2 \mu^{2}\left(1+2 \alpha_{0}\right) \alpha_{2}\right) k^{2} \\
& +2 \omega^{2} \alpha_{2} \mu^{2}+\omega^{2} \alpha_{1} \lambda \mu=0, \\
& \left(\frac{G^{\prime}}{G}\right)^{1}:\left(\left(-22 \mu \lambda^{2}-16 \mu^{2}-\lambda^{4}\right) \alpha_{1}\right. \\
& \left.-30 \mu \lambda \alpha_{2}\left(4 \mu+\lambda^{2}\right)\right) q k^{4} \\
& +\left(-6 \alpha_{1}^{2} \lambda \mu+\left(-2 \mu-\lambda^{2}-2 \alpha_{0} \lambda^{2}-4 \alpha_{0} \mu-12 \alpha_{2} \mu^{2}\right) \alpha_{1}\right. \\
& \left.-6 \mu \lambda \alpha_{2}\left(1+2 \alpha_{0}\right)\right) k^{2}+\omega^{2}\left(2 \mu+\lambda^{2}\right) \alpha_{1} \\
& +6 \omega^{2} \alpha_{2} \lambda \mu=0 \text {, } \\
& \left(\frac{G^{\prime}}{G}\right)^{2}:\left(-15 \lambda\left(4 \mu+\lambda^{2}\right) \alpha_{1}\right. \\
& \left.-8 \alpha_{2}\left(29 \mu \lambda^{2}+2 \lambda^{4}+17 \mu^{2}\right)\right) q k^{4} \\
& +\left(\left(-4 \lambda^{2}-8 \mu\right) \alpha_{1}^{2}-3 \lambda\left(2 \alpha_{0}+1+10 \mu \alpha_{2}\right) \alpha_{1}\right. \\
& \left.-4 \alpha_{2}\left(3 \alpha_{2} \mu^{2}+2 \mu+\lambda^{2}+4 \alpha_{0} \mu+2 \alpha_{0} \lambda^{2}\right)\right) k^{2} \\
& +3 \omega^{2} \alpha_{1} \lambda+4 \omega^{2} \alpha_{2}\left(2 \mu+\lambda^{2}\right)=0, \\
& \left(\frac{G^{\prime}}{G}\right)^{3}:\left(\left(-40 \mu-50 \lambda^{2}\right) \alpha_{1}-10 \lambda \alpha_{2}\left(13 \lambda^{2}+44 \mu\right)\right) q k^{4} \\
& +\left(-10 \alpha_{1}^{2} \lambda+\left(-36 \mu \alpha_{2}-18 \alpha_{2} \lambda^{2}-2-4 \alpha_{0}\right) \alpha_{1}\right. \\
& \left.-2 \alpha_{2} \lambda\left(14 \mu \alpha_{2}+5+10 \alpha_{0}\right)\right) k^{2} \\
& +2 \omega^{2} \alpha_{1}+10 \omega^{2} \alpha_{2} \lambda=0, \\
& \left(\frac{G^{\prime}}{G}\right)^{4}:\left(-60 \alpha_{1} \lambda-30 \alpha_{2}\left(11 \lambda^{2}+8 \mu\right)\right) q k^{4} \\
& +\left(-6 \alpha_{1}^{2}-42 \alpha_{2} \alpha_{1} \lambda\right. \\
& \left.-2 \alpha_{2}\left(16 \mu \alpha_{2}+8 \alpha_{2} \lambda^{2}+3+6 \alpha_{0}\right)\right) k^{2} \\
& +6 \omega^{2} \alpha_{2}=0 \\
& \left(\frac{G^{\prime}}{G}\right)^{5}:\left(-24 \alpha_{1}-336 \alpha_{2} \lambda\right) q k^{4} \\
& +\left(-36 \alpha_{2}^{2} \lambda-24 \alpha_{2} \alpha_{1}\right) k^{2}=0, \\
& \left(\frac{G^{\prime}}{G}\right)^{6}:-120 q k^{4} \alpha_{2}-10 k^{2} \alpha_{2}^{2}=0 \text {. }
\end{aligned}
$$

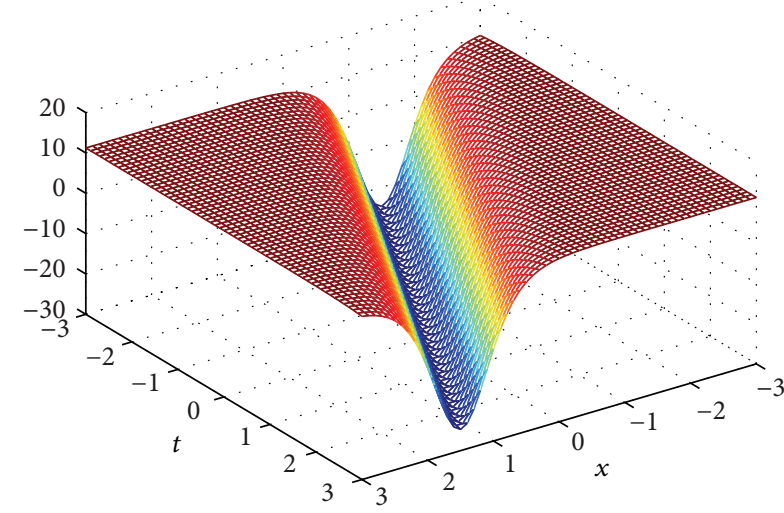

FIgURE 1: Hyperbolic function solution (20a) of the Boussinesq equation (5) for $q=-1, k=-1, \omega=1 / 2, \lambda=4, \mu=1$, and $\eta_{\mathscr{H}}=0$.

Solving the obtained algebraic equations by the use of Maple, we get the following results:

$$
\begin{gathered}
\left\{\alpha_{0}=-\frac{8 q k^{4} \mu+q k^{4} \lambda^{2}-\omega^{2}+k^{2}}{k^{2}},\right. \\
\left.\alpha_{1}=-12 k^{2} q \lambda, \alpha_{2}=-12 k^{2} q\right\},
\end{gathered}
$$

and $k, \omega, \lambda$, and $\mu$ are arbitrary constants. Therefore, substitute the previous case in (15), we get

$$
\begin{aligned}
U= & -12 k^{2} q\left(\frac{G^{\prime}}{G}\right)^{2}-12 k^{2} q \lambda\left(\frac{G^{\prime}}{G}\right) \\
& -\frac{8 q k^{4} \mu+q k^{4} \lambda^{2}-\omega^{2}+k^{2}}{k^{2}} .
\end{aligned}
$$

Substituting the general solutions (11) into (18), we obtain three types of traveling wave solutions of Boussinesq equation (5) in the view of the positive, negative, or zero of $\lambda^{2}-4 \mu$.

When $\mathscr{D}=\lambda^{2}-4 \mu>0$, we obtain hyperbolic function solution $U_{\mathscr{H}}$ of Boussinesq equation (5) (see Figure 1) as follows:

$$
\begin{aligned}
& U_{\mathscr{H}}(\xi) \\
& =-3 k^{2} q\left(\left(\left(\sqrt { \mathscr { D } } \left[C_{1} \sinh ((1 / 2) \sqrt{\mathscr{D}} \xi)\right.\right.\right.\right. \\
& \left.\left.+C_{2} \cosh ((1 / 2) \sqrt{\mathscr{D}} \xi)\right]\right) \\
& \times\left(C_{2} \sinh ((1 / 2) \sqrt{\mathscr{D}} \xi)\right. \\
& \left.\left.\left.\quad+C_{1} \cosh ((1 / 2) \sqrt{\mathscr{D}} \xi)\right)^{-1}\right)-\lambda\right)^{2}
\end{aligned}
$$




$$
\begin{gathered}
-6 k^{2} q \lambda\left(\left(\left(\sqrt { \mathscr { D } } \left[C_{1} \sinh ((1 / 2) \sqrt{\mathscr{D}} \xi)\right.\right.\right.\right. \\
\left.\left.+C_{2} \cosh ((1 / 2) \sqrt{\mathscr{D}} \xi)\right]\right) \\
\times\left(C_{2} \sinh ((1 / 2) \sqrt{\mathscr{D}} \xi)\right. \\
\left.\left.\left.+C_{1} \cosh ((1 / 2) \sqrt{\mathscr{D}} \xi)\right)^{-1}\right)-\lambda\right) \\
-\frac{8 q k^{4} \mu+q k^{4} \lambda^{2}-\omega^{2}+k^{2}}{k^{2}},
\end{gathered}
$$

where $\xi=k x+\omega t$, and $C_{1}, C_{2}$ are arbitrary constants. It is easy to see that the hyperbolic solution (19) can be rewritten at $C_{1}^{2}>C_{2}^{2}$ as follows:

$$
\begin{aligned}
u_{\mathscr{H}}(x, t)= & -3 k^{2} q \mathscr{D} \tanh ^{2}\left(\frac{1}{2} \sqrt{\mathscr{D}} \xi+\eta_{\mathscr{H}}\right) \\
& -\frac{-2 q k^{4} \mathscr{D}-\omega^{2}+k^{2}}{k^{2}},
\end{aligned}
$$

while at $C_{1}^{2}<C_{2}^{2}$, one can obtain

$$
\begin{aligned}
u_{\mathscr{C}}(x, t)= & -3 k^{2} q \mathscr{D} \operatorname{coth}^{2}\left(\frac{1}{2} \sqrt{\mathscr{D}} \xi+\eta_{\mathscr{H}}\right) \\
& -\frac{-2 q k^{4} \mathscr{D}-\omega^{2}+k^{2}}{k^{2}},
\end{aligned}
$$

where $\xi=k x+\omega t, \eta_{\mathscr{H}}=\tanh ^{-1}\left(C_{1} / C_{2}\right)$, and $k, \omega, \lambda$, and $\mu$ are arbitrary constants. Now, when $\mathscr{D}=\lambda^{2}-4 \mu<0$, the trigonometric function solutions $U_{\mathscr{T}}$ of Boussinesq equation (5) will be

$$
\begin{gathered}
U_{\mathscr{T}}(\xi) \\
=-3 k^{2} q\left(\left(\left(\sqrt { - \mathscr { D } } \left[-C_{1} \sin ((1 / 2) \sqrt{-\mathscr{D}} \xi)\right.\right.\right.\right. \\
\left.\left.+C_{2} \cos ((1 / 2) \sqrt{-\mathscr{D}} \xi)\right]\right) \\
\times\left(C_{2} \sin ((1 / 2) \sqrt{-\mathscr{D} \xi})\right. \\
\left.\left.\left.+C_{1} \cos ((1 / 2) \sqrt{-\mathscr{D} \xi})\right)^{-1}\right)-\lambda\right)^{2} \\
-6 k^{2} q \lambda\left(\left(\left(\sqrt { - \mathscr { D } } \left[-C_{1} \sin ((1 / 2) \sqrt{-\mathscr{D}} \xi)\right.\right.\right.\right. \\
\left.\left.+C_{2} \cos ((1 / 2) \sqrt{-\mathscr{D}} \xi)\right]\right) \\
\times\left(C_{2} \sin ((1 / 2) \sqrt{-\mathscr{D} \xi})\right. \\
\left.\left.\left.+C_{1} \cos ((1 / 2) \sqrt{-\mathscr{D}} \xi)\right)^{-1}\right)-\lambda\right) \\
-\frac{8 q k^{4} \mu+q k^{4} \lambda^{2}-\omega^{2}+k^{2}}{k^{2}}
\end{gathered}
$$

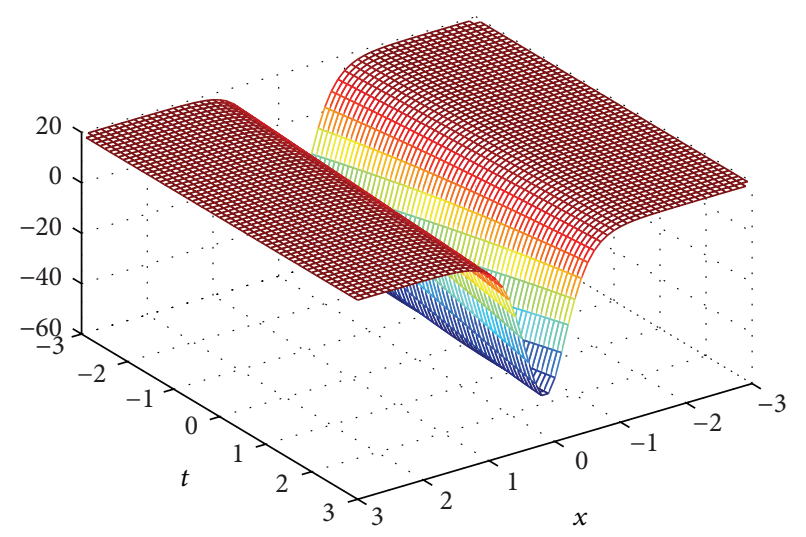

Figure 2: Trigonometric function solution (22a) of the Boussinesq equation (5) for $q=-1, k=-1, \omega=1 / 20, \lambda=4, \mu=-1$, and $\eta_{\mathscr{T}}=0$.

where $\xi=k x+\omega t$, and $C_{1}, C_{2}$ are arbitrary constants (see Figure 2). Similarly, the trigonometric solutions (21) can be rewritten at $C_{1}^{2}>C_{2}^{2}$ and $C_{1}^{2}<C_{2}^{2}$, respectively, as follows:

$$
\begin{aligned}
u_{\mathscr{T}}(x, t)= & 3 k^{2} q \mathscr{D} \tan ^{2}\left(\frac{1}{2} \sqrt{-\mathscr{D}} \xi+\eta_{\mathscr{T}}\right) \\
& -\frac{-2 q k^{4} \mathscr{D}-\omega^{2}+k^{2}}{k^{2}}, \\
u_{\mathscr{T}}(x, t)= & 3 k^{2} q \mathscr{D} \cot ^{2}\left(\frac{1}{2} \sqrt{-\mathscr{D}} \xi+\eta_{\mathscr{T}}\right) \\
& -\frac{-2 q k^{4} \mathscr{D}-\omega^{2}+k^{2}}{k^{2}},
\end{aligned}
$$

where $\xi=k x+\omega t, \eta_{\mathscr{T}}=\tan ^{-1}\left(C_{1} / C_{2}\right)$, and $k, \omega, \lambda$, and $\mu$ are arbitrary constants. Finally, when $\lambda^{2}-4 \mu=0$, then the rational function solutions to (5) will be

$$
u_{\mathrm{rat}}(x, t)=-\frac{12 k^{2} q C_{2}^{2}}{\left(C_{1}+C_{2}(k x+\omega t)\right)^{2}}+\frac{\omega^{2}}{k^{2}}-1,
$$

where $C_{1}, C_{2}, k$, and $\omega$ are arbitrary constants.

3.2. Improved Boussinesq Equation. Similar to the previous section, to obtain the traveling wave solution of improved Boussinesq equation (6) we substitute the gauge transformation (13) into (6) to obtain nonlinear ordinary differential equation

$$
\left(\omega^{2}-k^{2}\right) U^{\prime \prime}-k^{2}\left(\frac{1}{2} U^{2}+q \omega^{2} U^{\prime \prime}\right)^{\prime \prime}=0 .
$$

According to Step 1, we get $m+4=2 m+2$; hence, $m=2$. Then, similar to the previous section, we suppose that (24) has the same formal solutions (15). Substituting (15) along with (10) into (24) and collecting all terms with the same order of $\left(G^{\prime} / G\right)$ together, the left-hand sides of $(24)$ are converted into a polynomial in $\left(G^{\prime} / G\right)$. Setting each coefficient of each 
polynomial to zero, we derive a set of algebraic equations for $k, \omega, \lambda, \mu, \alpha_{0}, \alpha_{1}$, and $\alpha_{2}$ as follows:

$$
\begin{aligned}
& \left(\frac{G^{\prime}}{G}\right)^{0}:\left(\left(-\mu \omega^{2} \lambda\left(\lambda^{2}+8 \mu\right) \alpha_{1}-2 \mu^{2} \omega^{2} \alpha_{2}\left(8 \mu+7 \lambda^{2}\right)\right) q\right. \\
& \left.-2 \alpha_{1}^{2} \mu^{2}-\lambda \mu\left(1+2 \alpha_{0}\right) \alpha_{1}-2 \mu^{2} \alpha_{2}\left(1+2 \alpha_{0}\right)\right) k^{2} \\
& +2 \omega^{2} \alpha_{2} \mu^{2}+\omega^{2} \alpha_{1} \lambda \mu=0, \\
& \left(\frac{G^{\prime}}{G}\right)^{1}:\left(\left(-\omega^{2}\left(22 \lambda^{2} \mu+16 \mu^{2}+\lambda^{4}\right) \alpha_{1}\right.\right. \\
& \left.-30 \mu \omega^{2} \alpha_{2} \lambda\left(\lambda^{2}+4 \mu\right)\right) q-6 \alpha_{1}{ }^{2} \lambda \mu \\
& +\left(-2 \mu-\lambda^{2}-12 \alpha_{2} \mu^{2}-4 \alpha_{0} \mu-2 \alpha_{0} \lambda^{2}\right) \alpha_{1} \\
& \left.-6 \lambda \mu \alpha_{2}\left(1+2 \alpha_{0}\right)\right) k^{2} \\
& +\omega^{2}\left(2 \mu+\lambda^{2}\right) \alpha_{1}+6 \omega^{2} \alpha_{2} \lambda \mu=0 \\
& \left(\frac{G^{\prime}}{G}\right)^{2}:\left(\left(-15 \omega^{2} \lambda\left(\lambda^{2}+4 \mu\right) \alpha_{1}\right.\right. \\
& \left.-8 \omega^{2} \alpha_{2}\left(29 \lambda^{2} \mu+2 \lambda^{4}+17 \mu^{2}\right)\right) q \\
& +\left(-4 \lambda^{2}-8 \mu\right) \alpha_{1}^{2}-3 \lambda\left(1+2 \alpha_{0}+10 \alpha_{2} \mu\right) \alpha_{1} \\
& \left.-4 \alpha_{2}\left(3 \alpha_{2} \mu^{2}+2 \mu+\lambda^{2}+4 \alpha_{0} \mu+2 \alpha_{0} \lambda^{2}\right)\right) k^{2} \\
& +3 \omega^{2} \alpha_{1} \lambda+4 \omega^{2} \alpha_{2}\left(2 \mu+\lambda^{2}\right)=0, \\
& \left(\frac{G^{\prime}}{G}\right)^{3}:\left(\left(-10 \omega^{2}\left(4 \mu+5 \lambda^{2}\right) \alpha_{1}\right.\right. \\
& \left.-10 \omega^{2} \alpha_{2} \lambda\left(13 \lambda^{2}+44 \mu\right)\right) q-10 \alpha_{1}^{2} \lambda \\
& +\left(-4 \alpha_{0}-2-36 \alpha_{2} \mu-18 \alpha_{2} \lambda^{2}\right) \alpha_{1} \\
& \left.-2 \alpha_{2} \lambda\left(14 \alpha_{2} \mu+10 \alpha_{0}+5\right)\right) k^{2} \\
& +10 \omega^{2} \alpha_{2} \lambda+2 \omega^{2} \alpha_{1}=0, \\
& \left(\frac{G^{\prime}}{G}\right)^{4}:\left(\left(-60 \omega^{2} \alpha_{1} \lambda-30 \omega^{2} \alpha_{2}\left(11 \lambda^{2}+8 \mu\right)\right) q\right. \\
& -6 \alpha_{1}^{2}-42 \alpha_{2} \alpha_{1} \lambda \\
& \left.-2 \alpha_{2}\left(16 \alpha_{2} \mu+8 \alpha_{2} \lambda^{2}+3+6 \alpha_{0}\right)\right) k^{2} \\
& +6 \omega^{2} \alpha_{2}=0 \text {, } \\
& \left(\frac{G^{\prime}}{G}\right)^{5}:\left(\left(-24 \omega^{2} \alpha_{1}-336 \omega^{2} \alpha_{2} \lambda\right) q-36 \alpha_{2}^{2} \lambda-24 \alpha_{2} \alpha_{1}\right) k^{2} \\
& =0 \text {, } \\
& \left(\frac{G^{\prime}}{G}\right)^{6}:\left(-10 \alpha_{2}^{2}-120 q \omega^{2} \alpha_{2}\right) k^{2}=0
\end{aligned}
$$

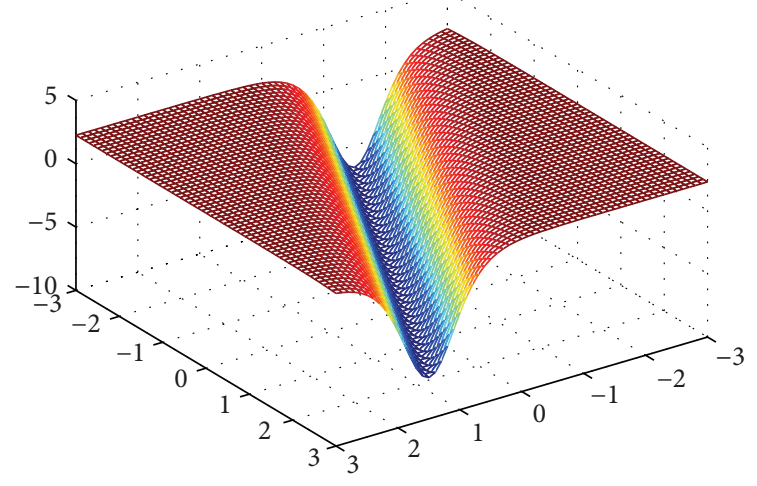

Figure 3: Hyperbolic function solution (20a) of the improved Boussinesq equation (6) for $q=-1, k=-1, \omega=1 / 2, \lambda=4, \mu=1$, and $\eta_{\mathscr{H}}=0$.

and solving by use of Maple, we get the following results:

$$
\begin{gathered}
\left\{\alpha_{0}=-\frac{8 q k^{2} \omega^{2} \mu+q k^{2} \omega^{2} \lambda^{2}+k^{2}-\omega^{2}}{k^{2}},\right. \\
\left.\alpha_{1}=-12 \omega^{2} q \lambda, \alpha_{2}=-12 \omega^{2} q\right\},
\end{gathered}
$$

and $k, \omega, \lambda$, and $\mu$ are arbitrary constants. Therefore, the solution (15) leads to

$$
\begin{aligned}
U= & -12 \omega^{2} q\left(\frac{G^{\prime}}{G}\right)^{2}-12 \omega^{2} q \lambda\left(\frac{G^{\prime}}{G}\right) \\
& -\frac{8 q k^{2} \omega^{2} \mu+q k^{2} \omega^{2} \lambda^{2}+k^{2}-\omega^{2}}{k^{2}}
\end{aligned}
$$

Now, for $\mathscr{D}=\lambda^{2}-4 \mu>0$ and $\mathscr{D}=\lambda^{2}-4 \mu<0$, the hyperbolic function solution $U_{\mathscr{H}}$, and trigonometric function solution $U_{\mathscr{T}}$, of improved Boussinesq equation (6) are obtained as follows (see Figures 3 and 4), respectively:

$$
\begin{aligned}
& U_{\mathscr{H}}(\xi) \\
& \begin{aligned}
=-3 \omega^{2} q( & \left(\left(\sqrt { \mathscr { D } } \left[C_{1} \sinh ((1 / 2) \sqrt{\mathscr{D}} \xi)\right.\right.\right. \\
& \left.\left.+C_{2} \cosh ((1 / 2) \sqrt{\mathscr{D}} \xi)\right]\right) \\
& \times\left(C_{2} \sinh ((1 / 2) \sqrt{\mathscr{D}} \xi)\right. \\
& \left.\left.\left.+C_{1} \cosh ((1 / 2) \sqrt{\mathscr{D}} \xi)\right)^{-1}\right)-\lambda\right)^{2}
\end{aligned}
\end{aligned}
$$




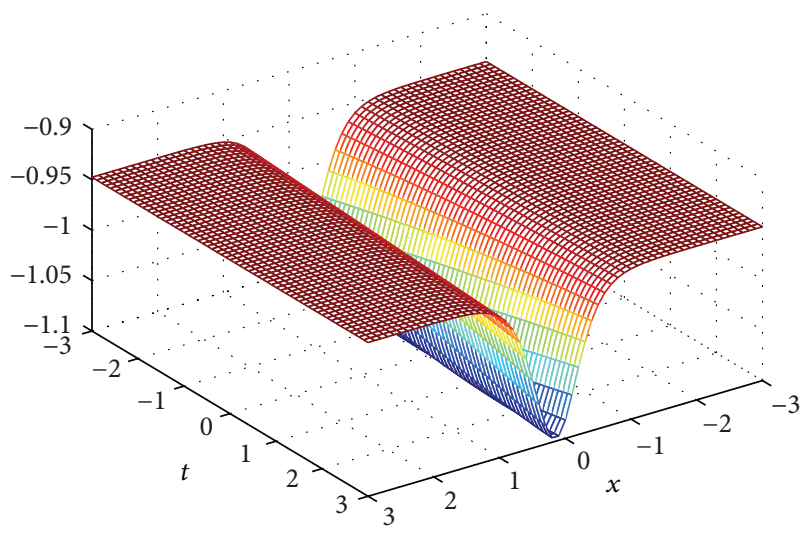

FIgURE 4: Trigonometric function solution (22a) of the improved Boussinesq equation (6) for $q=-1, k=-1, \omega=1 / 20, \lambda=4$, $\mu=-1$, and $\eta_{\mathscr{T}}=0$.

$$
\begin{gathered}
-6 \omega^{2} q \lambda\left(\left(\left(\sqrt { \mathscr { D } } \left[C_{1} \sinh ((1 / 2) \sqrt{\mathscr{D}} \xi)\right.\right.\right.\right. \\
\left.\left.+C_{2} \cosh ((1 / 2) \sqrt{\mathscr{D}} \xi)\right]\right) \\
\times\left(C_{2} \sinh ((1 / 2) \sqrt{\mathscr{D} \xi})\right. \\
\left.\left.\left.+C_{1} \cosh ((1 / 2) \sqrt{\mathscr{D}} \xi)\right)^{-1}\right)-\lambda\right) \\
-\frac{8 q k^{2} \omega^{2} \mu+q k^{2} \omega^{2} \lambda^{2}+k^{2}-\omega^{2}}{k^{2}},
\end{gathered}
$$

$$
\begin{gathered}
U_{\mathscr{T}}(\xi) \\
=-3 \omega^{2} q\left(\left(\left(\sqrt { - \mathscr { D } } \left[-C_{1} \sin ((1 / 2) \sqrt{-\mathscr{D}} \xi)\right.\right.\right.\right. \\
\left.\left.+C_{2} \cos ((1 / 2) \sqrt{-\mathscr{D}} \xi)\right]\right) \\
\times\left(C_{2} \sin ((1 / 2) \sqrt{-\mathscr{D} \xi})\right. \\
\left.\left.\left.+C_{1} \cos ((1 / 2) \sqrt{-\mathscr{D} \xi})\right)^{-1}\right)-\lambda\right)^{2} \\
-6 \omega^{2} q \lambda\left(\left(\left(\sqrt { - \mathscr { D } } \left[-C_{1} \sin ((1 / 2) \sqrt{-\mathscr{D}} \xi)\right.\right.\right.\right. \\
\left.\left.+C_{2} \cos ((1 / 2) \sqrt{-\mathscr{D}} \xi)\right]\right) \\
\times\left(C_{2} \sin ((1 / 2) \sqrt{-\mathscr{D} \xi})\right. \\
\left.\left.\left.+C_{1} \cos ((1 / 2) \sqrt{-\mathscr{D}} \xi)\right)^{-1}\right)-\lambda\right) \\
-\frac{8 q k^{2} \omega^{2} \mu+q k^{2} \omega^{2} \lambda^{2}+k^{2}-\omega^{2}}{k^{2}}
\end{gathered}
$$

where $\xi=k x+\omega t$, and $C_{1}, C_{2}$ are arbitrary constants. It is easy to see that the hyperbolic solution (28) and trigonometric solution (29) can be rewritten at $C_{1}^{2}>C_{2}^{2}$ as follows:

$$
\begin{aligned}
u_{\mathscr{H}}(x, t)= & -3 \omega^{2} q \mathscr{D} \tanh ^{2}\left(\frac{1}{2} \sqrt{\mathscr{D}} \xi+\eta_{\mathscr{H}}\right) \\
& -\frac{-2 q k^{2} \omega^{2} \mathscr{D}-\omega^{2}+k^{2}}{k^{2}}, \\
u_{\mathscr{T}}(x, t)= & 3 \omega^{2} q \mathscr{D} \tan ^{2}\left(\frac{1}{2} \sqrt{-\mathscr{D}} \xi+\eta_{\mathscr{T}}\right) \\
& -\frac{-2 q k^{2} \omega^{2} \mathscr{D}-\omega^{2}+k^{2}}{k^{2}},
\end{aligned}
$$

while at $C_{1}^{2}<C_{2}^{2}$, one can obtain

$$
\begin{aligned}
u_{\mathscr{H}}(x, t)= & -3 \omega^{2} q \mathscr{D} \operatorname{coth}^{2}\left(\frac{1}{2} \sqrt{\mathscr{D}} \xi+\eta_{\mathscr{H}}\right) \\
& -\frac{-2 q k^{2} \omega^{2} \mathscr{D}-\omega^{2}+k^{2}}{k^{2}}, \\
u_{\mathscr{T}}(x, t)= & 3 \omega^{2} q \mathscr{D} \cot ^{2}\left(\frac{1}{2} \sqrt{-\mathscr{D}} \xi+\eta_{\mathscr{T}}\right) \\
& -\frac{-2 q k^{2} \omega^{2} \mathscr{D}-\omega^{2}+k^{2}}{k^{2}},
\end{aligned}
$$

where $\xi=k x+\omega t, \eta_{\mathscr{H}}=\tanh ^{-1}\left(C_{1} / C_{2}\right), \eta_{\mathscr{T}}=\tan ^{-1}\left(C_{1} / C_{2}\right)$, and $k, \omega, \lambda$, and $\mu$ are arbitrary constants.

Finally, when $\lambda^{2}-4 \mu=0$, then the rational function solutions of improved Boussinesq equation (6) will be

$$
u_{\text {rat }}(x, t)=-\frac{12 \omega^{2} q C_{2}^{2}}{\left(C_{1}+C_{2}(k x+\omega t)\right)^{2}}+\frac{\omega^{2}}{k^{2}}-1,
$$

where $C_{1}, C_{2}, k$, and $\omega$ are arbitrary constants.

\section{Conclusions}

This study shows that the $\left(G^{\prime} / G\right)$-expansion method is quite efficient and practically well suited for use in finding exact solutions for the Boussinesq equation and improved Boussinesq equations. The reliability of the method and the reduction in the size of computational domain give this method a wider applicability. Though the obtained solutions represent only a small part of the large variety of possible solutions for the equations considered, they might serve as seeding solutions for a class of localized structures existing in the physical systems. Furthermore, our solutions are in more general forms, and many known solutions to these equations are only special cases of them. With the aid of Maple, we have assured the correctness of the obtained solutions by putting them back into the original equation.

\section{Conflict of Interests}

This paper does not have any conflict of interest with the authors research topics. 


\section{Acknowledgment}

The first author would like to thank the Young Researchers and Elite Club, Islamic Azad University, Ardabil Branch for its financial support.

\section{References}

[1] J. S. Russell, "Report on waves," in Proceedings of the 14th Meeting of the British Association for the Advancement of Science, 1844.

[2] M. J. Ablowtiz and J. F. Ladik, "On the solution of a class of nonliear partial difference equations," Studies in Applied Mathematics, vol. 57, pp. 1-12, 1977.

[3] J. Boussinesq, "Thorie de l'intumescence liquide, applele onde solitaire ou de translation, se propageant dans un canal rectangulaire," Comptes Rendus de l'Académie des Sciences, vol. 72, pp. 755-759, 1871.

[4] J. Boussinesq, "Thorie des ondes et des remous qui se propagent le long d'un canal rectangulaire horizontal, en communiquant au liquide contenu dans ce canal des vitesses sensiblement pareilles de la surface au fond," Journal de Mathématiques Pures et Appliquées, vol. 17, pp. 55-108, 1872.

[5] O. V. Kaptsov, "Construction of exact solutions of the Boussinesq equation," Journal of Applied Mechanics and Technical Physics, vol. 39, no. 3, pp. 389-392, 1998.

[6] I. L. Bogolubsky, "Some examples of inelastic soliton interaction," Computer Physics Communications, vol. 13, no. 3, pp. 149$155,1977$.

[7] V. G. Makhankov, "Dynamics of classical solitons (in nonintegrable systems)," Physics Reports, vol. 35, no. 1, pp. 1-128, 1978.

[8] J. L. Bona and R. L. Sachs, "Global existence of smooth solutions and stability of solitary waves for a generalized Boussinesq equation," Communications in Mathematical Physics, vol. 118, no. 1, pp. 15-29, 1988.

[9] P. A. Clarkson, "New exact solutions of the Boussinesq equation," European Journal of Applied Mathematics, vol. 1, no. 3, pp. 279-300, 1990.

[10] R. Hirota, "Solutions of the classical boussinesq equation and the spherical boussinesq equation: the Wronskian technique," Journal of the Physical Society of Japan, vol. 55, no. 7, pp. 21372150, 1986.

[11] Y. Liu, "Instability and blow-up of solutions to a generalized Boussinesq equation," SIAM Journal on Mathematical Analysis, vol. 26, no. 6, pp. 1527-1546, 1995.

[12] N. Yajima, "On a growing mode of the Boussinesq equation," Progress of Theoretical Physics, vol. 69, no. 2, pp. 678-680, 1983.

[13] A. Nakamura, "Exact solitary wave solutions of the spherical boussinesq equation," Journal of the Physical Society of Japan, vol. 54, no. 11, pp. 4111-4114, 1985.

[14] S. B. Wang and G. W. Chen, "Small amplitude solutions of the generalized IMBq equation," Journal of Mathematical Analysis and Applications, vol. 274, no. 2, pp. 846-866, 2002.

[15] M. Wang, X. Li, and J. Zhang, "The $\left(\operatorname{frac}\left(G^{\prime} / G\right)\right)$-expansion method and travelling wave solutions of nonlinear evolution equations in mathematical physics," Physics Letters A, vol. 372, no. 4, pp. 417-423, 2008.

[16] R. Abazari, “The $\left(G^{\prime} / G\right)$-expansion method for the coupled Boussinesq equation," Procedia Engineering,, vol. 10, pp. 28452850, 2011.
[17] E. M. E. Zayed and K. A. Gepreel, “The $\left(G^{\prime} / G\right)$-expansion method for finding traveling wave solutions of nonlinear partial differential equations in mathematical physics," Journal of Mathematical Physics, vol. 50, no. 1, Article ID 013502, 12 pages, 2009.

[18] Y. He, S. Li, and Y. Long, "Exact solutions of the KudryashovSinelshchikov equation using the multiple $\left(G^{\prime} / G\right)$-expansion method," Mathematical Problems in Engineering, vol. 2013, Article ID 708049, 7 pages, 2013.

[19] A. Kilıcman and R. Abazari, "Travelling wave solutions of the Schrödinger-Boussinesq system," Abstract and Applied Analysis, vol. 2012, Article ID 198398, 11 pages, 2012. 


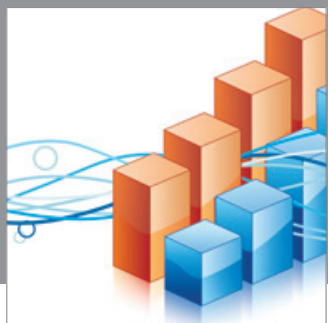

Advances in

Operations Research

mansans

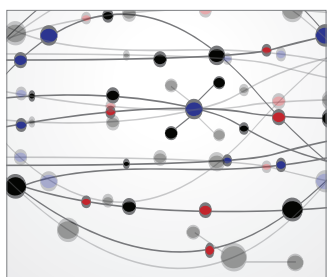

The Scientific World Journal
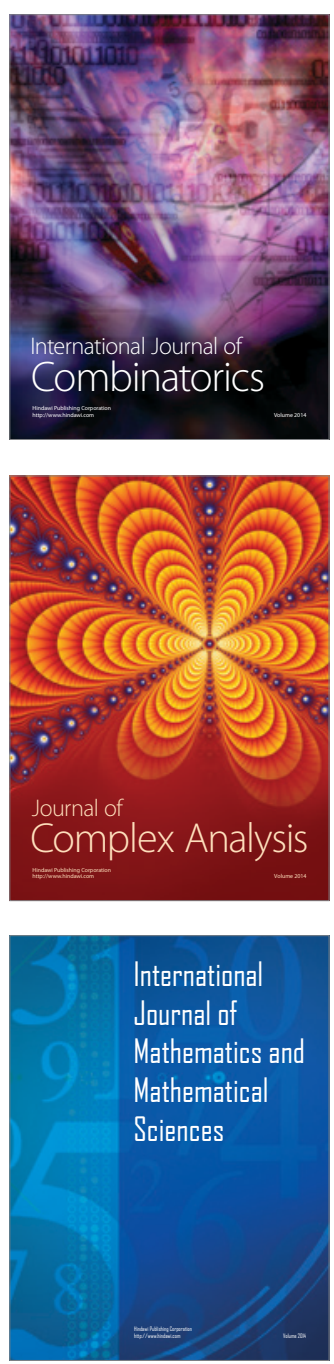
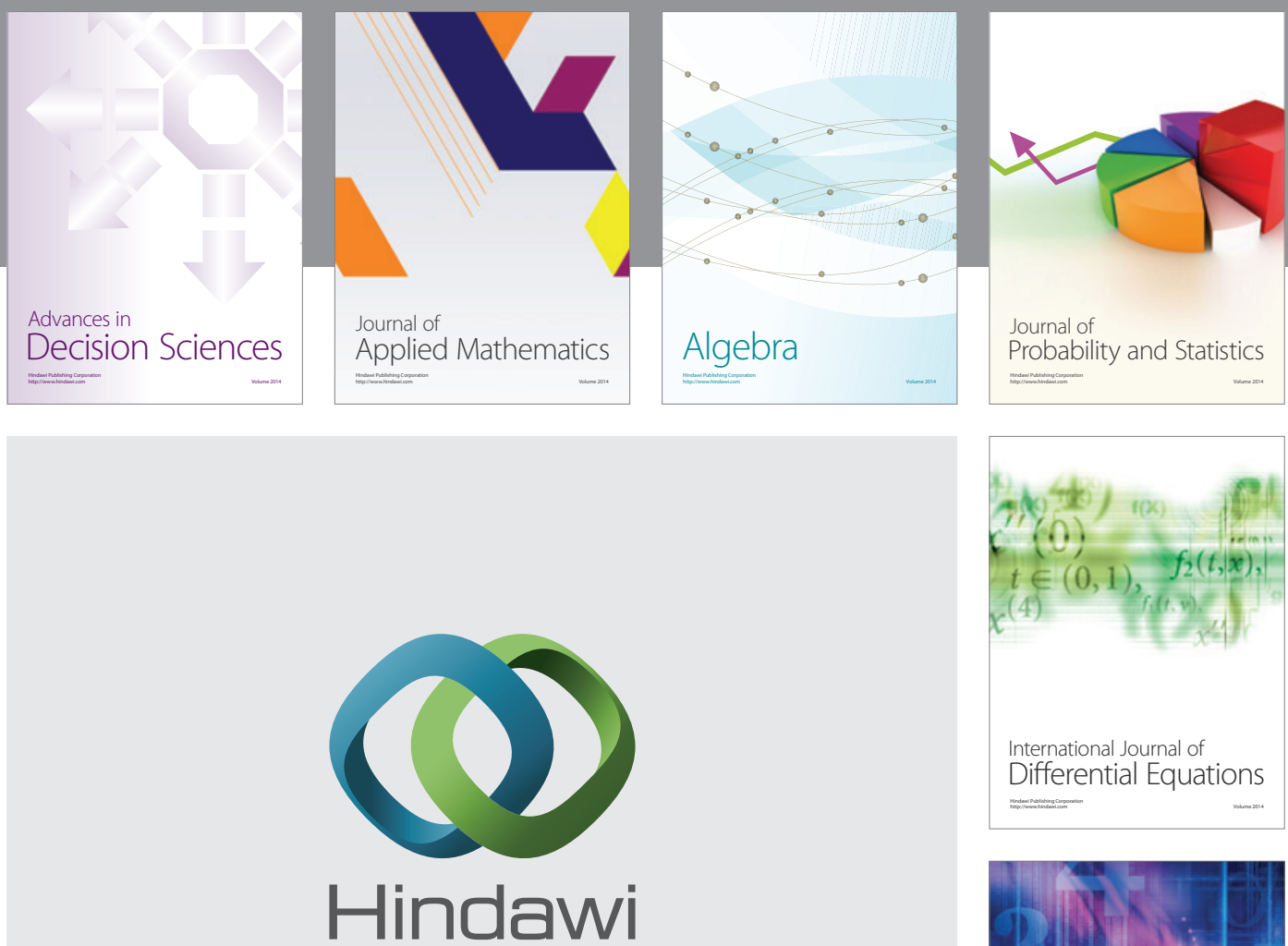

Submit your manuscripts at http://www.hindawi.com
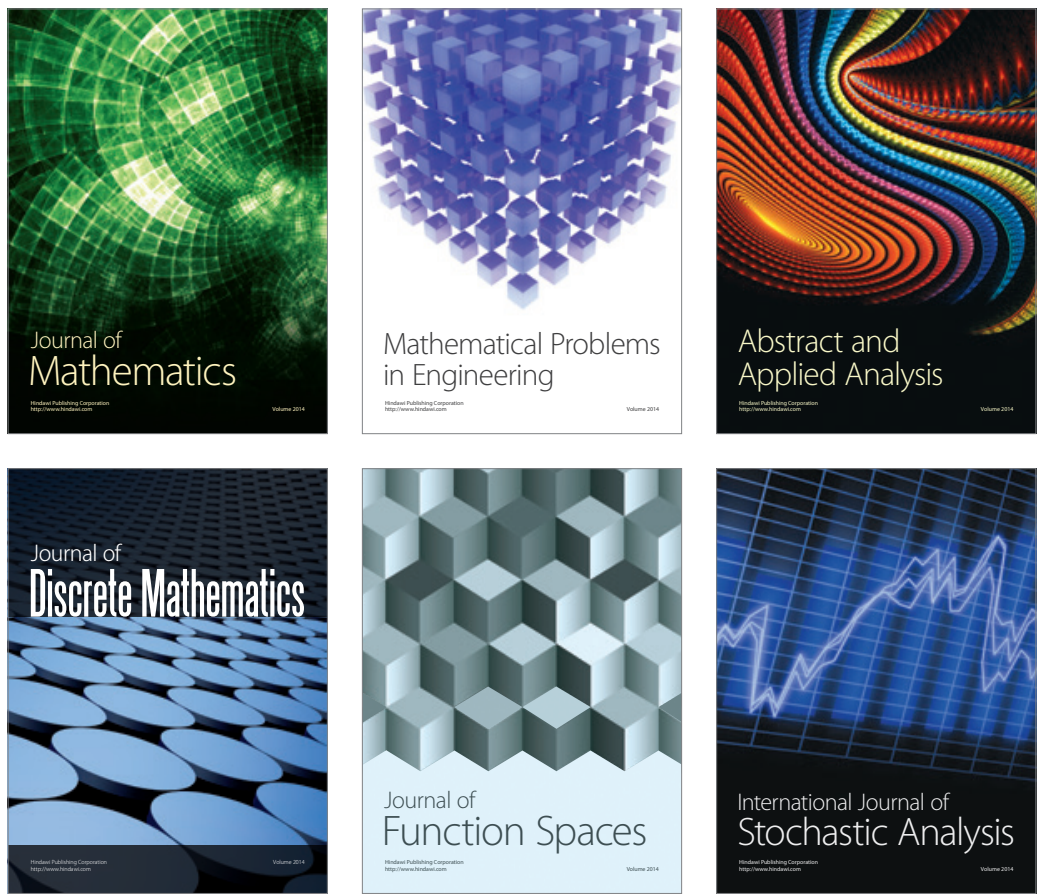

Journal of

Function Spaces

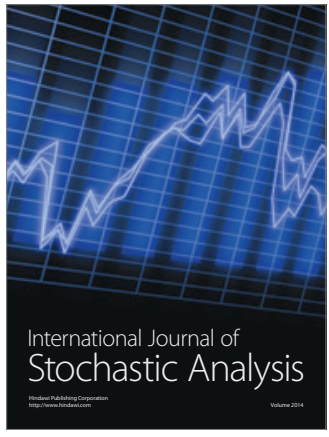

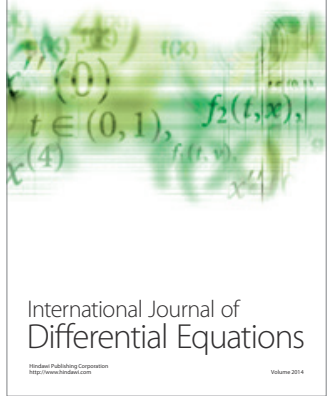
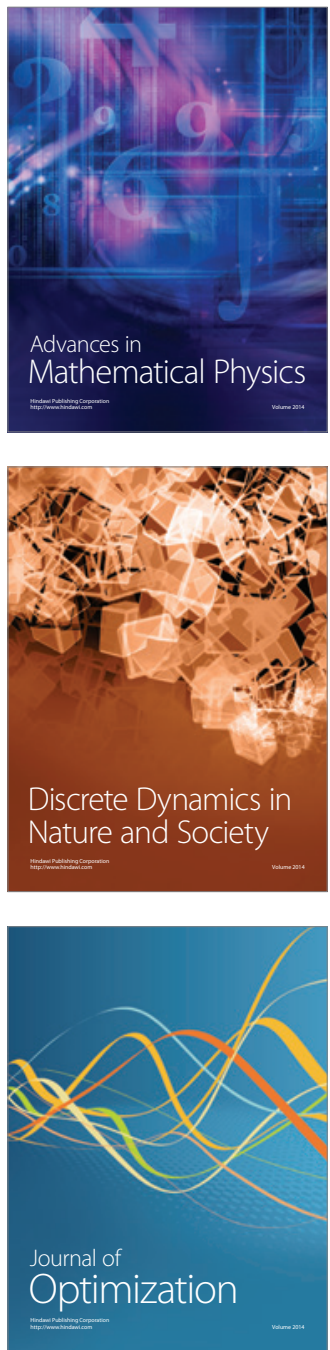